

\title{
Blockade of Experimental Multiple Sclerosis by Inhibition of the Acid Sphingomyelinase/Ceramide System
}

\author{
Katrin Anne Becker ${ }^{a}$ Ramona Halmer ${ }^{b}$ Laura Davies $^{b}$ Brian D. Henry ${ }^{a, c}$ \\ Regan Ziobro-Henry ${ }^{a, c}$ Yann Decker ${ }^{b}$ Yang Liu ${ }^{b}$ Erich Gulbins ${ }^{a, c} \quad K^{2}$ laus Fassbender ${ }^{b}$ \\ Silke Walter ${ }^{b}$
}

aDept. of Molecular Biology, University of Duisburg-Essen, Essen, Germany; ${ }^{b}$ Dept. of Neurology, University Hospital of the Saarland, Homburg/Saar, Germany; 'Dept. of Surgery, University of Cincinnati, Cincinnati, $\mathrm{OH}$, USA

\section{Key Words}

Multiple sclerosis - Acid sphingomyelinase - Ceramide - Tight junctions - Experimental autoimmune encephalomyelitis

\begin{abstract}
Background: Multiple sclerosis (MS) is a severe and common autoimmune disorder of the central nervous system. Despite the availability of several novel treatment options, the disease is still poorly controlled, since the pathophysiological mechanisms are not fully understood. Methods: We tested the role of the acid sphingomyelinase/ceramide system in a model of MS, i.e. experimental autoimmune encephalomyelitis (EAE). Mice were immunized with myelin-oligodendrocyte glycoprotein and the development of the disease was analyzed by histology, immunological tests and clinical assessment in wildtype and acid sphingomyelinase (Asm)-deficient mice. Results: Genetic deficiency of acid sphingomyelinase (Asm) protected against clinical symptoms in EAE and markedly attenuated the characteristic detrimental neuroinflammatory response. T lymphocyte adhesion, integrity of tight junctions, bloodbrain barrier disruption and subsequent intracerebral infiltration of inflammatory cells were blocked in Asm-deficient mice after immunization. This resulted in an almost complete block of the development of disease symptoms in these mice, while wildtype mice showed severe neurological symptoms typical for EAE. Conclusion: Activation of the Asm/ceramide system is a central step for the development of EAE. Our findings may serve to identify novel therapeutic strategies for MS patients.

\section{Introduction}

Multiple sclerosis (MS) is one of the most important autoimmune disorders and is the leading cause of neurological disability among young adults in the Western world [1,2]. The main

Dr. Silke Walter, and Dr. Katrin Anne Becker

KARGER
Dept. of Neurology, University Hospital of the Saarland, Kirrberger Strasse, 66421 Homburg/Saar, (Germany); and Dept. of Molecular Biology, University of DuisburgEssen, Hufelandstrasse 55, 45122 Essen, (Germany); E-Mail silke.walter@uks.eu / katrin. anne.becker@uni-due.de 
pathological feature of MS is inflammatory infiltration of the central nervous system (CNS), which leads to extensive demyelination, neuronal loss, and gliotic scars that are responsible for neurological symptoms such as motor and sensory deficits or impairment of vision. Active inflammatory lesions are characterized by an initial infiltration of $\mathrm{CD}^{+}$and $\mathrm{CD}^{+} \mathrm{T}$ cells $[1,3,4]$, which orchestrate an inflammatory effector phase that ultimately leads to brain damage [5].

The adhesion of lymphocytes to endothelial cells and the migration of lymphocytes from the peripheral circulation across the blood-brain barrier (BBB) are crucial steps in the pathophysiology of MS. Lymphocyte adhesion and transendothelial migration are complex multi-step processes involving interactions between complementary adhesion molecules on the surfaces of lymphocytes and endothelial cells $[6,7]$. Once $T$ cells have attached to endothelial cells, the tight junctions of the $\mathrm{BBB}$ are disrupted, and $\mathrm{T}$ lymphocytes migrate into the parenchyma of the CNS, where they interact with resident inflammatory cells [8-10]. This interaction leads to a complex cascade with the production of vasoactive substances, chemokines, and cytotoxic and myelinotoxic cytokines. Further peripheral stimulation of leukocytes and their migration into the CNS are responsible for progressive damage to brain tissue [11-13].

Although some of the pathophysiological events leading to MS have been characterized, the cause of the disease is presently still unknown, and therapeutic options remain limited. All of the currently used immunomodulatory drugs have been shown to reduce the number of MS relapses; however, none of them does completely protect against disease progression. Therefore, the identification of novel targets and the development of new therapies are of utmost importance for the treatment of MS patients.

Acid sphingomyelinase (Asm) is a ubiquitously expressed protein that has been shown to be critically involved in cellular stress responses $[14,15]$. Asm hydrolyses sphingomyelin to ceramide. Ceramide has been shown to be involved in many stress signals [16], in the induction of cell death [17] and, in particular, inflammation [18]. Thus, it was demonstrated that the Asm/ceramide system determines inflammation in the lung of cystic fibrosis mice $[19,20]$. The system is also involved in the inflammatory reaction seen in Crohn's disease [21], a severe inflammatory disease of the intestine. The Asm/ceramide system also serves to cluster surface receptors and thereby permits and amplifies their signal transduction [22, 23].

Here, we report that the Asm/ceramide system plays a central role in the development of experimental autoimmune encephalomyelitis (EAE), an animal model of MS. Deficiency of the Asm protects mice from development of EAE. Asm-deficiency prevents BBB leakage and tight junction disruption, leukocyte adhesion to endothelial cells and thereby protects from leukocyte immigration into the CNS and neuroinflammatory damage with associated clinical symptoms.

\section{Material and Methods}

Mice

All animal experiments were performed in compliance with the German Guide for the Care and Use of Laboratory Animals. All studies were approved by the Landesamt für Gesundheit und Verbraucherschutz, Saarbruecken (approval numbers: 34/2008; 41/2009; 33/2009; 19/2012; 06/2013). Female 6 to 8 week-old Asmdeficient mice (protein, Asm; gene symbol, Smpd1) on a C57BL/6J genetic background and wild-type (wt) female littermates were obtained from our breeding facility, where they were kept in a pathogen-free environment according to the Federation of Laboratory Animal Science Associations (FELASA) criteria. The genotype of Asmdeficient mice was confirmed by polymerase chain reaction (PCR) before experimentation.

Induction of active EAE

EAE is the most widely studied animal model of MS [24]. Mice with the C57BL/6J background were immunized independent from their cycle phase with $300 \mu \mathrm{g}$ myelin oligodendrocytic glycoprotein $\left(\mathrm{MOG}_{\text {aa35-55}}\right.$; Charité Medical University, Berlin, Germany). MOG $_{\text {aa35-55 }}$ was emulsified 1:1 (v/v) in incomplete Freund's adjuvant (IFA; DIFCO, BD Bioscience, Heidelberg, Germany) supplemented with $4 \mathrm{mg} / \mathrm{mL}$ non-viable, desiccated Mycobacterium tuberculosis (H37RA; DIFCO, BD Bioscience) and were injected subcutaneously into the axillary and inguinal 
Becker et al.: Acid Sphingomyelinase in Experimental Multiple Sclerosis

lymph node regions. On days 0 and 2 after injection, $300 \mathrm{ng}$ pertussis toxin (Axxora, Lörrach, Germany) was injected intraperitoneally (ip). Clinical disease was scored daily as follows: 0.5 , limp tail; 1 , hind leg weakness; 2 , hind leg paresis; 3 , hind leg paraparesis and incontinence; 4 , tetraparesis.

\section{Histopathologic assessment}

Mice were perfused with $4 \%$ paraformaldehyde (PFA) administered through the left ventricle of the heart. The brain and spinal cord were removed and were snap-frozen in Tissue Tek (Sakura Finetek; Hartenstein, Würzburg, Germany) in melting isopentane with liquid nitrogen. The frozen tissue was cut serially into 6 - $\mu \mathrm{m}$ sections with a cryostat microtome (Leica Microsystems, Wetzlar, Germany), air-dried overnight, and fixed with acetone at $-20^{\circ} \mathrm{C}$. Frozen serial sections were subjected to immunohistochemical analysis with a three-step immunoperoxidase technique in a humidified chamber. The following monoclonal antibodies were used: anti-CD4, anti-CD8, anti-

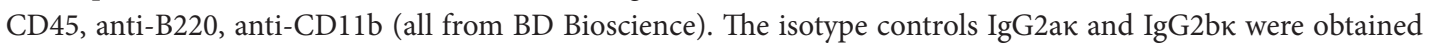
from IQ Products (Houston, TX). Sections were incubated, in 30-minute steps with phosphate-buffered saline (PBS) washes between each step, with the primary antibody of interest followed by a biotinylated secondary goat anti-rat IgG (Vectastain; Linearis, Wertheim-Bettingen, Germany) and finally by a horseradish peroxidaseconjugated streptavidin (Vectastain; Linearis). Sections were developed with $0.07 \%$ aminoethylcarbazole (AEC; Sigma-Aldrich) and $0.009 \%$ hydrogen peroxide in $0.01 \mathrm{~mol} / \mathrm{L}$ acetate buffer $(\mathrm{pH} 5.2)$ for 10 minutes and then counterstained with hematoxylin-eosin and cover-slipped with Aquatex (Merck, Darmstadt, Germany).

For detection of tight junctions, mice were perfused with 4\% PFA, and sections of the spinal cord were obtained as described above and embedded in paraffin. Paraffin-embedded sections were dewaxed; washed in $\mathrm{H}_{2} \mathrm{O}$; blocked in HEPES/saline (H/S; $132 \mathrm{mM} \mathrm{NaCl}, 20 \mathrm{mM}$ HEPES [pH 7.4], $5 \mathrm{mM} \mathrm{KCl,} 1 \mathrm{mM} \mathrm{CaCl}, 0.7 \mathrm{mM} \mathrm{MgCl}_{2}$, $0.8 \mathrm{mM} \mathrm{MgSO}_{4}$ ) supplemented with 5\% fetal calf serum (FCS); washed in PBS; incubated with Pepsin (Invitrogen, Karlsruhe, Germany) for $10 \mathrm{~min}$; washed in PBS; and incubated with anti-claudin-5 antibody (Invitrogen), antiZO-1 antibody (Invitrogen) or isotype control antibodies (Invitrogen) for $45 \mathrm{~min}$ in H/S + 5\% FCS. Samples were washed 3 times in PBS supplemented with $0.5 \%$ Tween 20 and once in PBS and then incubated for 45 min with Cy3-conjugated $\mathrm{F}(\mathrm{ab})_{2}$ fragments of donkey-anti-mouse IgG (Jackson Laboratories, Dianova, Hamburg, Germany) or Cy3-conjugated $\mathrm{F}(\mathrm{ab})_{2}$ fragments of donkey-anti-rabbit IgG (Jackson Laboratories), both 1:1000 in H/S + 5\% FCS. Samples were washed an additional 3 times in PBS/0.05\% Tween 20 and once in PBS, embedded in Mowiol, and analyzed by confocal microscopy.

Evans blue dye (12.5 mg/kg; Fluka, Sigma-Aldrich) was administered iv to healthy mice and to EAEimmunized Asm-deficient and wt littermate mice at the peak of disease; the dye was allowed to circulate for 4 hours before the mice were sacrificed. The mice were then perfused with PBS administered through the left ventricle. The brain was fixed overnight in 4\% PFA and then snap-frozen in Tissue Tek, after which $30-\mu \mathrm{m}$ sections were cut with a cryostat microtome, air-dried, and fixed with acetone for 2 minutes. The sections were allowed to air-dry, dipped in xylol, and cover-slipped with entellan (Merck).

Frozen brain sections from wildtype and Asm-deficient mice were fixed in acetone for 10 min at room temperature, washed 3 times in PBS, blocked in PBS supplemented with 5\% FCS, washed twice in PBS and stained overnight with FITC-Isolectin (1:50, Vector Laboratories). The samples were washed 3 times in PBS supplemented with $0.05 \%$ Tween 20, once in PBS and stained with anti-VCAM-1 (1:100, BD-Bioscience) or anti-ICAM-1 (1:100, Biolegend) antibodies for $45 \mathrm{~min}$ at room temperature. Samples were washed again in PBS/0.05\% Tween 20 and stained with Cy3-coupled secondary antibodies (1:500, Jackson Immunoresearch). Samples were washed again in PBS/0.05\% Tween 20, once in PBS, embedded in mowiol and analysed on a Leica SP5 confocal microscope. The fluorescence intensity of anti-VCAM-1 and anti-ICAM-1 stainings was determined in FITC-isolectin-positive endothelial cells. FITC-isolectin served to identify endothelial cells. 30-50 endothelial cells were analyzed per brain.

\section{Lymphocyte adhesion assay}

16-well chamber slides (VWR International, Darmstadt, Germany) were coated with $50 \mu \mathrm{g} / \mathrm{mL}$ fibronectin (Roche, Mannheim, Germany) and then plated with $2 \times 10^{4}$ bEnd. 3 cells per well (ATCC; LGS Standards, Wesel, Germany) for 2 days before the experiment. T-lymphocytes were obtained from EAE-immunized mice on day 14 after immunization by homogenizing axillary inguinal lymph nodes in Hank's Balanced Salt Solution (HBSS)/10\% FCS/25 mM HEPES solution and straining them through a $70-\mu \mathrm{m}$ cell filter. Cell suspensions were centrifuged and resuspended in migration assay medium (Dulbecco's Modified Eagle Medium [DMEM]/2\% GlutaMAX/1\% penicillin-streptomycin/25 mM HEPES/5\% FCS). T cells $\left(3 \times 10^{5}\right.$ cells per well) were co-incubated with the endothelial cells for 40 minutes at $4^{\circ} \mathrm{C}$ on a rocking shaker. 
Becker et al.: Acid Sphingomyelinase in Experimental Multiple Sclerosis

After co-incubation, non-adherent $\mathrm{T}$ cells were washed off with PBS, and the slides were fixed in $2.5 \%$ glutaraldehyde for 2 hours. After the fixation period, assays were counted with a light microscope. The following $\mathrm{T}$ cell treatments were tested: 30 -min preincubation at $37^{\circ} \mathrm{C}, 5 \% \mathrm{CO}_{2}$ with $20 \mu \mathrm{g} / \mathrm{mL}$ anti- $\alpha_{4}$-integrin antibody (CD49d; Millipore, Merck), with $20 \mu \mathrm{g} / \mathrm{mL}$ anti- $\beta_{1}$-integrin antibody (CD29; BioLegend, Biozol, Eching, Germany), with 20 $\mu \mathrm{g} / \mathrm{mL}$ of the respective isotype control, rat IgG2 ${ }_{\text {bkappa }}$ or armenian hamster IgG (BioLegend), or with $1 \mathrm{U} / \mathrm{mL}$ Asm (Sigma-Aldrich); or 5-min preincubation with $1 \mathrm{mM} \mathrm{Mn}^{2+}$ (Sigma-Aldrich). All experiments were performed in duplicate in the presence of $10 \mu \mathrm{M} \mathrm{ZnCl}_{2}$ (VWR International).

\section{Flow cytometry analysis}

For analysis of lymphocyte phenotypes and inflammatory cytokine production, the following conjugated monoclonal antibodies were used: anti-CD4 FITC, anti-CD8a PerCP, anti- $\beta_{1}$-integrin PE, anti- $\beta_{7}$-integrin PE, antiINF $\gamma$ PE, anti-interleukin (IL)-2 PE, anti-IL-4 PE (all from BD Pharmingen), and anti-IL-17 APC (eBioscience, Frankfurt, Germany). For intracellular cytokine staining, $2 \times 10^{5}$ cells were stimulated for 4 h with $500 \mathrm{ng} / \mathrm{mL}$ ionomycin (Sigma-Aldrich), $50 \mathrm{ng} / \mathrm{mL}$ phorbol 12-myristate 13-acetate (Sigma-Aldrich), and $10 \mu \mathrm{g} / \mathrm{mL}$ brefeldin A (eBioscience). After being washed, cell membranes were permeabilized with $0.1 \%$ saponin (Sigma-Aldrich). Cells were incubated with the respective antibody for $40 \mathrm{~min}$ in the dark at $4^{\circ} \mathrm{C}$. Samples were analyzed by flow cytometry (FACS Canto II BD) with FACS Diva Software (Becton Dickinson, Heidelberg, Germany).

\section{Matrix metalloproteinase detection}

Lymphocytes were obtained from 3 EAE-immunized Asm-deficient mice and 3 wt littermates on day 14 after immunization as described above. Two days before the assay, $1 \times 10^{5} \mathrm{bEnd} .3$ cells were plated in 24 -well plates, which were coated with $50 \mu \mathrm{g} / \mathrm{mL}$ fibronectin for $40 \mathrm{~min}$. At the time of the assay, $5 \times 10^{5} \mathrm{~T}$ cells per well were coincubated with the bEnd.3 monolayer. Supernatants were collected after 12 and 24 hours for detection of matrix metalloproteinases (MMPs) by enzyme-linked immunosorbent assay (ELISA). MMP7 (Blue Gene, Shanghai, China), and MMP9 (R\&D) concentrations were detected according to the manufacturer's instructions.

\section{Proliferation assays}

On day 14 after immunization, lymphocytes were isolated from lymph nodes and cultured in a 96-well plate $\left(2 \times 10^{5}\right.$ cells per well) in restimulation medium (RPMI medium 1640/10\% FCS/2\% GlutaMAX/1\% sodium pyruvate/1\% MEM/1\% penicillin-streptomycin/ $5 \times 10^{-5} \mathrm{M} \beta$-mercaptoethanol) supplemented with either $2.5 \mu \mathrm{g} / \mathrm{mL}$ Concanavalin A (Sigma-Aldrich) or MOG antigen (Charité Medical University). The cells were cultured for $48 \mathrm{~h}$ at $37^{\circ} \mathrm{C}$. After incubation, cells were pulsed with ${ }^{3} \mathrm{H}$-thymidine $(1 \mu \mathrm{Ci}$ per well) and incubated for an additional $16 \mathrm{~h}$ at $37^{\circ} \mathrm{C}$. Cells were then transferred onto a filter membrane for measurement of tritiated thymidine by a liquid scintillation counter. Means and SDs were calculated from duplicate or triplicate wells.

\section{Statistical analysis}

All data are displayed as mean \pm SD. Statistical analysis was performed with Student $t$ test or ANOVA, as appropriate. For analysis of disease frequency the chi-square test was applied. Statistical significance was defined as $\mathrm{p}<0.05$.

\section{Results}

\section{Asm deficiency protects against EAE}

To determine whether Asm plays a role in the development of EAE, we immunized Asmdeficient and C57BL/6 littermate (wt) mice with myelin oligodendrocyte glycoprotein (MOG) ${ }_{35-55^{\circ}}$ The wt animals exhibited clinical symptoms from day $15 \pm 2$, starting with limp tail and hind-leg paresis (Fig. 1A), which deteriorated to a clinical score of 3 to 4 (mean severity, $2.3 \pm 1.5$ ) by day 18 to 20 after immunization. In marked contrast, all Asm-deficient animals exhibited no or very mild clinical symptoms, with a maximum limp tail score of 0.5 , even after prolonged observation (up to day 35 after immunization) (Fig. 1A). The clinical resistance of Asm-deficient mice to EAE was associated with the absence of inflammatory infiltrations in brain and spinal cord, as determined by immunostaining, whereas wt mice exhibited prominent cerebral and spinal infiltration with $\mathrm{CD}^{+}$lymphocytes, B cells, and macrophages in the CNS after the induction of EAE (Fig. 1B). In 
Fig. 1. Genetic deficiency of acid sphingomyelinase (Asm) prevents active experimental autoimmune encephalomyelitis (EAE). Mice were immunized with myelin oligodendrocytic glycoprotein $\left(\mathrm{MOG}_{35-55}\right)$ in complete Freund's adjuvant (CFA). (A) Animals were evaluated daily for clinical symptoms. Asm-deficiency prevents EAE. (B) Histological analysis of brain and spinal cord demonstrated a massive inflammation in wild-type (wt) mice but not in Asm-deficient mice. Shown are means \pm SD of each $10 \mathrm{wt}$ and Asm-deficient mice in A. ${ }^{*}, \mathrm{p} \leq 0.05$. The histological pictures are representative of 13 wt and 12 Asm-deficient mice. Scale bar, $50 \mu \mathrm{m}$. Stainings with isotype control antibodies were all negative (not shown).

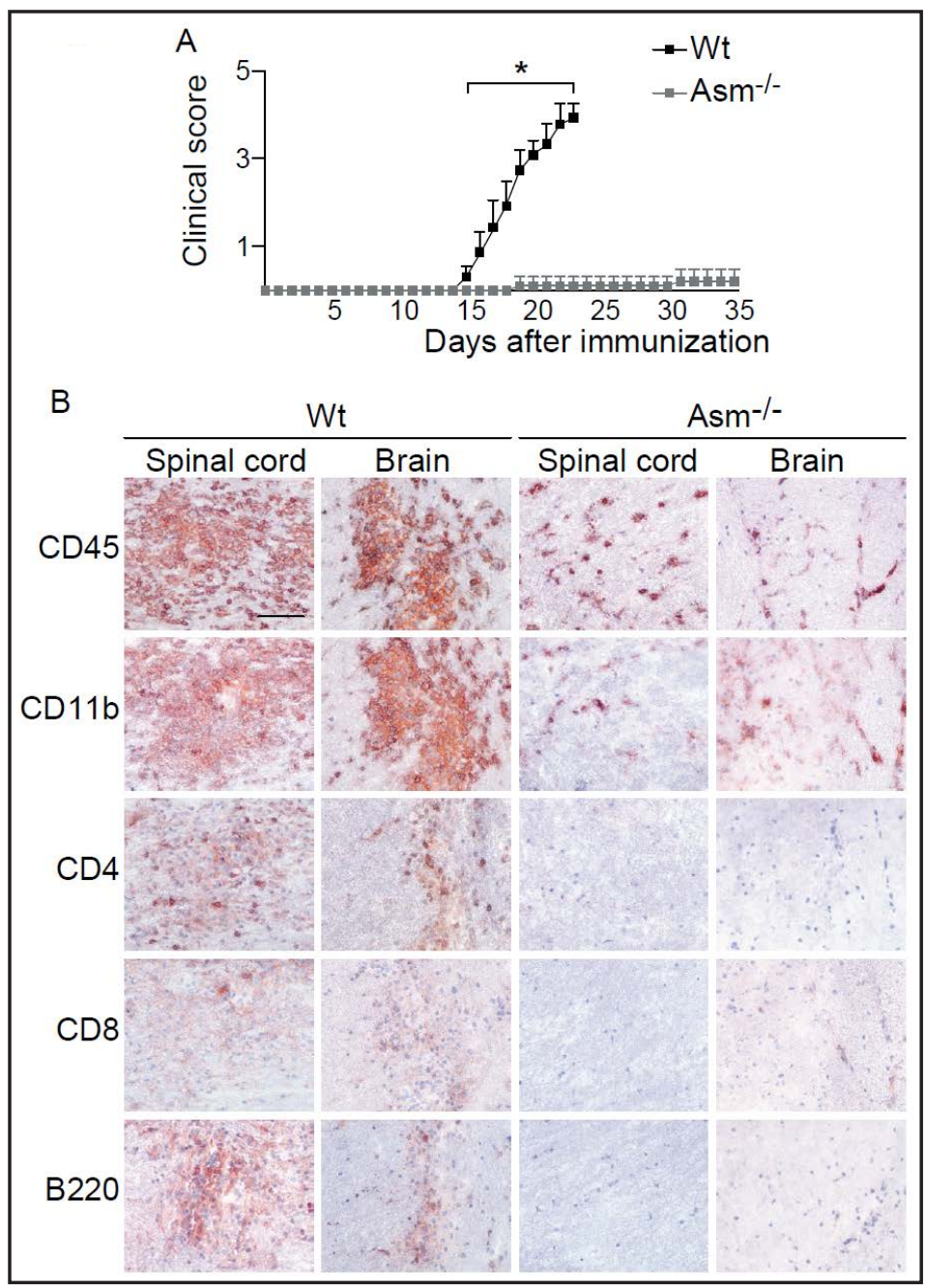

contrast to the lack of immigration of leukocyte into the CNS of Asm-deficient mice, these mice showed a strong microglial activation indicated by the positive staining for CD45 and CD11b as sign for the immune activation.

Taken together, these genetic findings demonstrate that Asm plays a key role in the development of the clinical and histopathological features of EAE. Deficiency of Asm prevents the development of EAE.

\section{Asm deficiency preserves the integrity of the blood-brain barrier}

To learn, which molecular mechanisms prevent EAE in Asm-deficient mice, we aimed to determine whether the typical EAE-induced breakdown of the BBB is altered in these mice. To this end, we injected Evans blue dye into EAE-immunized Asm-deficient and wt mice. Histological analysis of the brain demonstrated massive intraparenchymal leakage of Evans blue dye in wt mice but not in Asm-deficient mice (Fig. 2A). Moreover, tight junctions remained intact in EAEimmunized Asm-deficient mice but were disrupted in wt mice, as indicated by the degradation of claudin-5 and ZO-1 (Fig. 2B).

\section{Asm does not affect lymphocyte proliferation and antigen-mediated activation}

Antigen-specific proliferation and T-cell activation are crucial steps in the development of EAE. To determine whether Asm controls these processes, we used a ${ }^{3} \mathrm{H}$-thymidine assay to examine the proliferation capability of Asm-deficient lymphocytes derived from EAE-immunized mice. We observed no difference in MOG-induced proliferation between Asm-deficient and wt cells (Fig. 3A). Furthermore, we detected neither a difference in the expression levels of the 

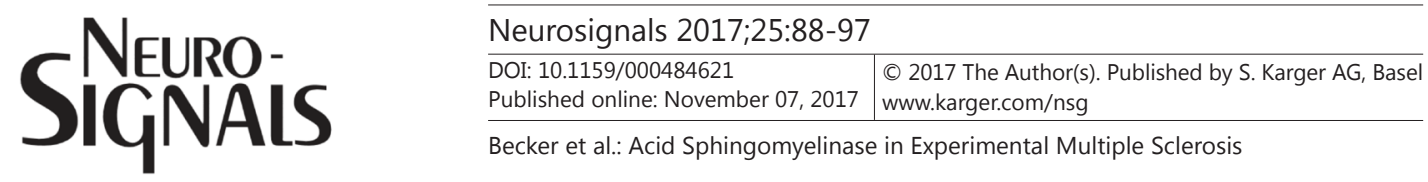

Fig. 2. Acid sphingomyelinase (Asm) and ceramide control tight-junction integrity in experimental autoimmune encephalomyelitis (EAE). Fluorescence microscopy of brain sections shows Evans Blue leakage (A) and disruption of the typical tight-junction and adherens proteins claudin-5 and ZO-1 (B) in wild-type (wt) but not in asm-deficient mice after induction of EAE. Shown are representative results of $5 \mathrm{wt}$ and 6 Asm-deficient mice. Scale bar, $100 \mu \mathrm{m}$.

Becker et al.: Acid Sphingomyelinase in Experimental Multiple Sclerosis

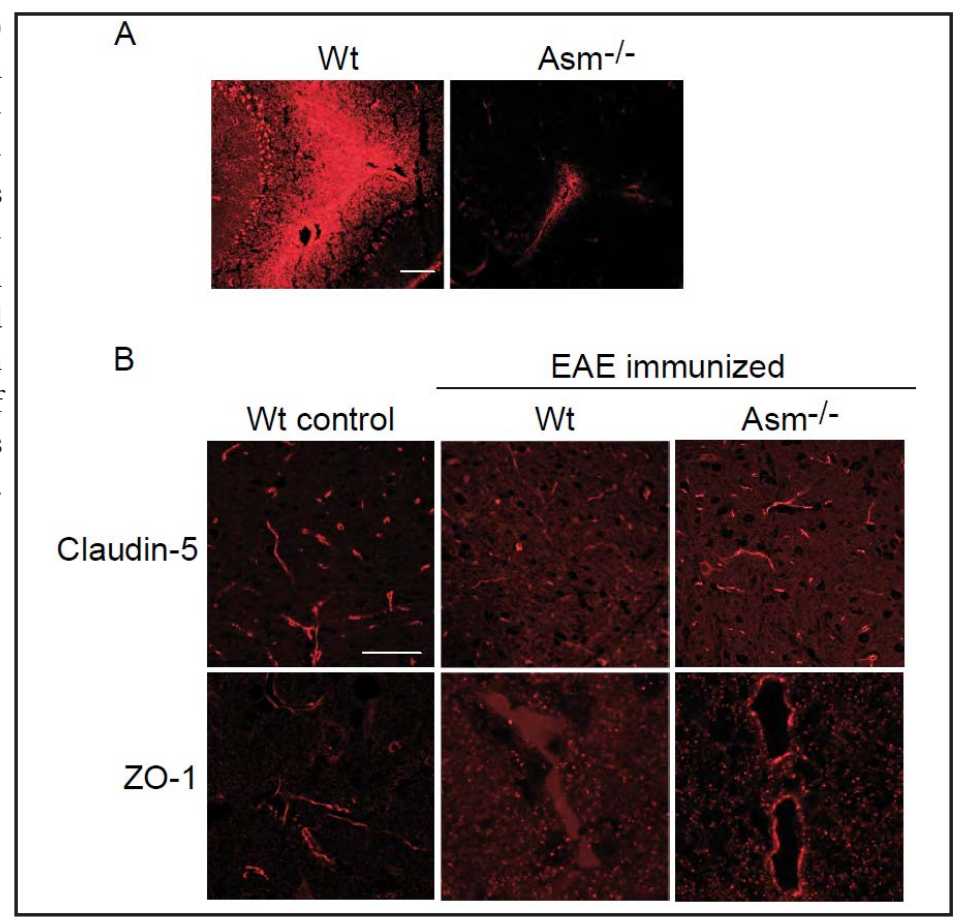

Fig. 3. Acid sphingomyelinase (Asm) does not influence T-cell proliferation, adhesion molecule expression or cytokine release after immunization. Deficiency of Asm does not affect proliferation $(n=7)$ (A) or expression levels of $\beta_{1}$-integrin $\left(\beta_{1}\right)$ or $\beta_{7}$-integrin $\left(\beta_{7}\right)$ in experimental autoimmune encephalomyelitis (EAE) lymphocytes ( $n=6)(B)$.

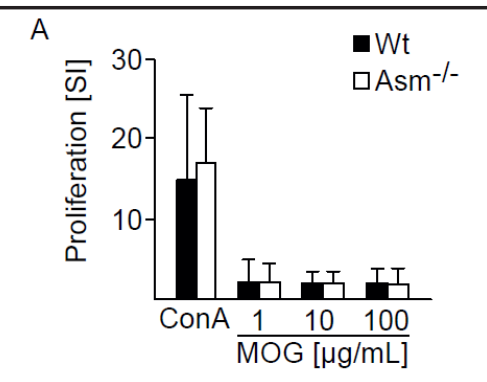

B
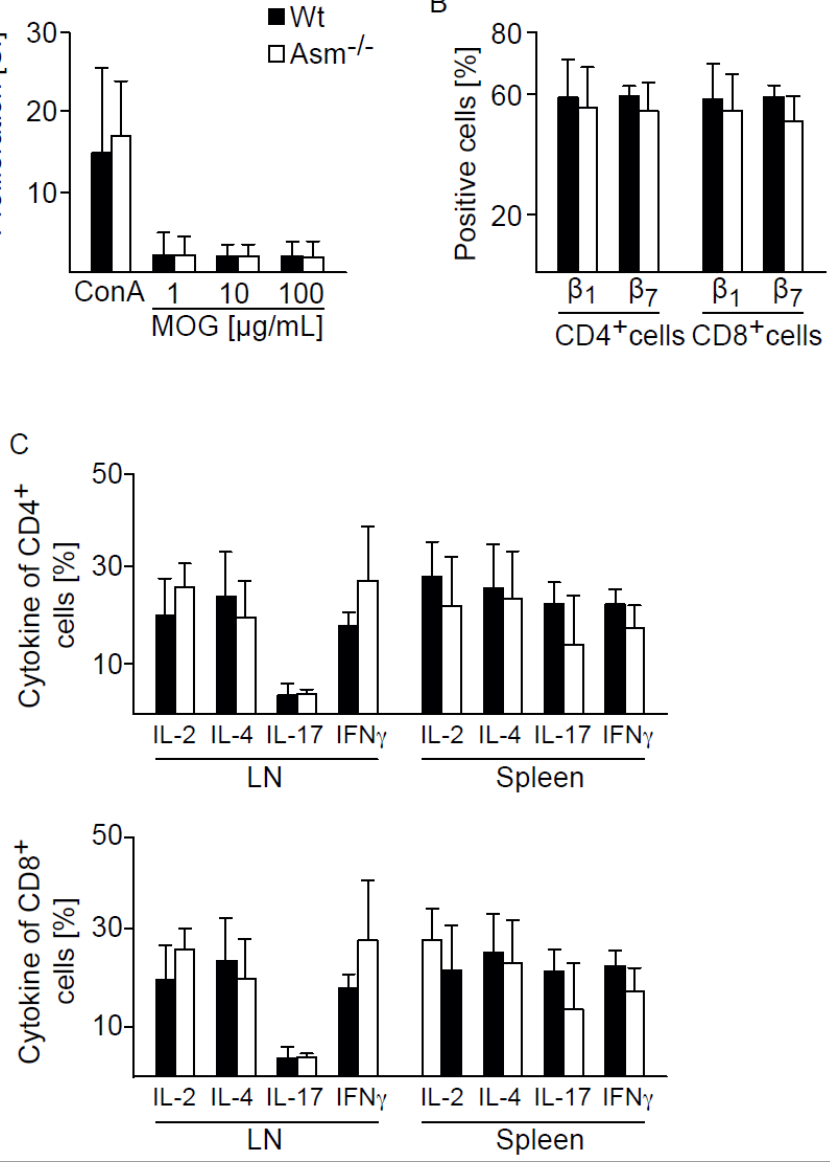

adhesion molecules $\beta_{1}$ - and $\beta_{7}$-integrin on lymphocytes nor a difference in the production of inflammatory cytokines (IL-2, IL-4, IL-17, IFN $\gamma$ ) by $\mathrm{CD}^{+}$or $\mathrm{CD}^{+} \mathrm{T}$ cells from lymph nodes of 


\section{SiGínAis}

Neurosignals 2017;25:88-97

\begin{tabular}{l|l}
\hline DOI: 10.1159/000484621 & (C) 2017 The Author(s). Published by S. Karger AG, Base
\end{tabular}

Published online: November 07, 2017 www.karger.com/nsg

Fig. 4. Acid sphingomyelinase (Asm) is required for expression of adhesion molecules in experimental autoimmune encephalomyelitis (EAE) brains and adhesion of EAE lymphocytes to endothelial cells. (A, B) Staining of brain sections with FITC-isolectin and Cy3-coupled anti-VCAM-1 or anti-ICAM-1 reveals a high expression of the adhesion molecules in endothelial cells from wildtype (wt) mice, while VCAM-1 and ICAM- 1 are reduced by $\sim 50 \%$ in brains from Asm-deficient mice. Displayed are typical examples (A) and the quantitative analysis from $8 \mathrm{wt}$ and 12 Asm-deficient mice (B). In each section we quantified the fluorescence in 30-50 endothelial cells. (C) In vitro adhesion assays show that the adhesion Asm-deficient lymphocytes to bEnd.3 cells is significantly lower than that of wt lymphocytes $(n=17)$. Treatment with purified Asm restores adhesion of Asm-deficient lymphocytes $(\mathrm{n}=10)$. The integrin activator $\mathrm{Mn}^{2+}$ restores adherence of Asm-deficient lymphocytes $(\mathrm{n}=10)$. Blockade with anti- $\alpha_{4}$-integrin antibody $(\mathrm{n}=14)$ or anti- $\beta_{1}$-integrin antibody $(n=3)$ reduces the adhesion of wt EAE-lym-

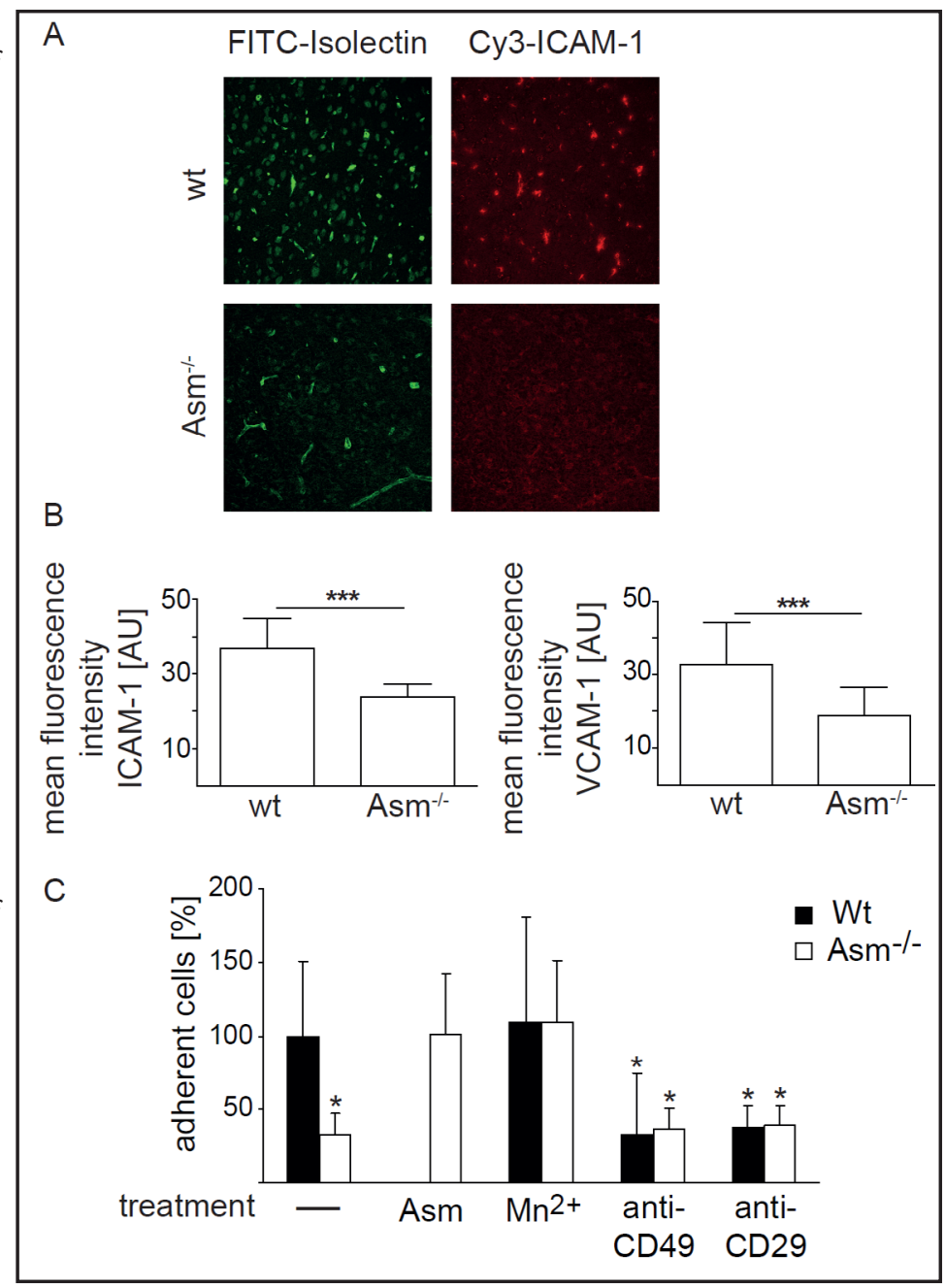

phocytes to the level of the residual adhesion of Asm-deficient EAE lymphocytes. The blocking antibodies do not further reduce the residual adhesion of Asm-deficient lymphocytes $(n=3)$. Shown are means $\pm S D ;{ }^{*}, \mathrm{p} \leq 0.05$.

Asm-deficient or wt mice after cell stimulation (Fig. 3B, C). Finally, we also found no difference in the release of matrix metalloproteinases, required for cell migration over the glia limitans [25, 26], in the supernatant of EAE-derived wt or Asm-deficient lymphocytes incubated on a bEnd.3 monolayer (not shown).

Asm-deficiency reduces expression of adhesion molecules in endothelial cells of the CNS and integrin-mediated adhesion of lymphocytes to endothelial cells

Next, we analyzed the expression of adhesion molecules on endothelial cells, i.e. intercellular adhesion molecule-1 (ICAM-1) and vascular cell adhesion molecule-1 (VCAM-1) in brain sections of immunized wt and Asm-deficient mice. These results indicate that deficiency of Asm reduced the expression of adhesion molecules on endothelial cells after immunization strengthening the notion that deficiency of Asm impairs activation and permeabilization of the BBB (Fig. 4A, B).

Endothelial adhesion of lymphocytes and associated transendothelial migration through the BBB is a complex pathophysiological step in the development of EAE or MS [6]. To determine whether Asm-deficiency also affects leukocyte-endothelial cell interactions, we performed in vitro adhesion assays using the bEnd.3 endothelial cell line to model the BBB. Adhesion of lymphocytes from Asm-deficient EAE-immunized mice to bEnd. 3 cells was $68 \%$ lower than that of wt EAElymphocytes (Fig. 4C). In turn, restoration of Asm in Asm-deficient lymphocytes re-established 
adhesion capability to that of wt levels (Fig. 4C). Previously, adhesion of self-reactive lymphocytes to endothelial cells of the BBB was shown to occur via $\alpha_{4} / \beta_{1}$-integrin [27]. Thus, we incubated Asmdeficient lymphocytes with manganese $\left(\mathrm{Mn}^{2+}\right)$, which directly activates integrins, to determine whether $\mathrm{Mn}^{2+}$ restores the adhesion of Asm-deficient lymphocytes. The results showed that $\mathrm{Mn}^{2+}$ bypasses Asm-deficiency and completely restores the adhesion of Asm-deficient lymphocytes to endothelial cells (Fig. 4C). Moreover, treating Asm-deficient lymphocytes with either anti- $\alpha_{4}^{-}$ integrin antibody or anti- $\beta_{1}$-integrin antibody did not further reduce the adhesion of Asm-deficient lymphocytes, whereas both antibodies reduced the adhesion of wt lymphocytes by $64-68 \%$, to the level of Asm-deficient lymphocytes (Fig. 4C).

\section{Discussion}

The results of this study demonstrate that genetic deficiency of the Asm/ceramide system strongly protects against EAE as experimental model of MS. Genetic Asm-deficiency prevents the disease symptoms. Thereby, the detrimental pathophysiological events initiated by immunization and characterized by adhesion of lymphocytes to endothelial cells, disruption of BBB and tight junctions, migration of T lymphocytes through the BBB and finally intracerebral inflammatory cell recruitment, causing EAE symptoms is blocked. The Asm/ceramide system controls inflammatory infiltration of the brain, and its inhibition prevents MS. This finding is of clinical importance, because inhibition of the Asm/ceramide system can provide complete protection from experimental MS.

Endothelial adhesion of leukocytes and subsequent transmigration through the BBB into the CNS are hallmarks in MS pathophysiology. Ceramide has been previously shown to mediate cell adhesion to endothelial cells [28]. These studies indicated that ceramide-enriched membrane platforms cluster adhesion molecules such as $\beta_{1}$-integrin [28] or ICAM-1 [29]. Receptor clustering results in a very high local density of cognate receptor molecules, thereby permitting and greatly amplifying signal transduction by this particular receptor. Such a fundamental molecular function of ceramide might explain its pleiotropic role in several physiological and pathological conditions. Although a lack of leukocyte adhesion might be a potential mechanism to prevent EAE, it remains to be determined whether such a lack of lymphocyte adhesion is the true mechanism how deficiency of the Asm prevents development of EAE.

Our studies indicate a central role of the Asm/ceramide system in pathophysiology of EAE. However, at present it remains to be determined whether the observed changes in wildtype mice such as leukocyte adhesion, disruption of the BBB, leukocyte immigration into the CNS and neuroinflammation mediate the resistance of Asm-deficient mice or whether they are readouts for a molecular defect in Asm-deficient mice that acts upstream/earlier in the pathophysiological cascade of EAE. As a limitation of this study, we did not adjust for female mice cycle phase, although an influence on motor symptoms has been described [30]. However the study results were reproduced in two different laboratories, so that we consider our results as robust.

In summary, the results of this study demonstrate a central role of Asm and ceramide in MS pathophysiology. Genetic deficiency of Asm blocks several events that are critical for EAE development, thereby preventing the clinical symptoms of this neuroinflammatory disease. Thus, inhibitors of the Asm might be used for treatment of MS, a concept that needs to be tested in future studies.

\section{Acknowledgments}

We thank Andrea Schottek, Nadine Niebergall, and Manuela Gries for excellent experimental support. The study was supported by DFG grant FA $212 / 8-2$ to KF and SW and by DFG grant Gu 335/29-1 to EG and GRK 2098 to KBF.

\section{Disclosure Statement}

The authors declare to have no conflict of interest. 


\section{References}

1 Noseworthy JH, Lucchinetti C, Rodriguez M, Weinshenker BG: Multiple sclerosis. New England J Med 2000;343:938-952.

2 Warnke C, Kieseier BC, Hartung HP: Biotherapeutics for the treatment of multiple sclerosis: hopes and hazards. J Neural Transmission 2013; 120:S55-60.

3 Traugott U, Reinherz EL, Raine CS: Multiple sclerosis: distribution of T cell subsets within active chronic lesions. Science 1983;219:308-310.

4 Hauser SL, Bhan AK, Gilles F, Kemp M, Kerr C, Weiner HL: Immunohistochemical analysis of the cellular infiltrate in multiple sclerosis lesions. Ann Neurol 1986;19:578-587.

5 Prineas JW, Wright RG: Macrophages, lymphocytes, and plasma cells in the perivascular compartment in chronic multiple sclerosis. Lab Invest 1978;38:409-421.

6 Engelhardt B, Ransohoff RM: Capture, crawl, cross: the T cell code to breach the blood-brain barriers. Trends Immunol 2012;33:579-589.

7 Kawakami N, Bartholomaus I, Pesic M, Mues M: An autoimmunity odyssey: how autoreactive T cells infiltrate into the CNS. Immunol Rev 2012;248:140-155.

8 Plumb J, McQuaid S, Mirakhur M, Kirk J: Abnormal endothelial tight junctions in active lesions and normalappearing white matter in multiple sclerosis. Brain Pathol 2002;12:154-169.

9 Kirk J, Plumb J, Mirakhur M, McQuaid S: Tight junctional abnormality in multiple sclerosis white matter affects all calibres of vessel and is associated with blood-brain barrier leakage and active demyelination. J Pathol 2003;201:319-327.

10 Mandel I, Paperna T, Glass-Marmor L, Volkowich A, Badarny S, Schwartz I, Vardi P, Koren I, Miller A.Tight junction proteins expression and modulation in immune cells and multiple sclerosis. Cell Mol Mede 2012;16:765775.

11 Imitola J, Chitnis T, Khoury SJ: Insights into the molecular pathogenesis of progression in multiple sclerosis: potential implications for future therapies. Arch Neurology 2006;63:25-33.

12 Frohman EM, Filippi M, Stuve O, Waxman SG, Corboy J, Phillips JT, Lucchinetti C, Wilken J, Karandikar N, Hemmer B, Monson N, De Keyser J, Hartung H, Steinman L, Oksenberg JR, Cree BA, Hauser S, Racke MK: Characterizing the mechanisms of progression in multiple sclerosis: evidence and new hypotheses for future directions. Arch Neurol 2005;62:1345-1356.

13 Odoardi F, Sie C, Streyl K, Ulaganathan VK, Schläger C, Lodygin D, Heckelsmiller K, Nietfeld W, Ellwart J, Klinkert WE, Lottaz C, Nosov M, Brinkmann V, Spang R, Lehrach H, Vingron M, Wekerle H, Flügel-Koch C, Flügel A: T cells become licensed in the lung to enter the central nervous system. Nature 2012;488:675-679.

14 Tullman MJ: A review of current and emerging therapeutic strategies in multiple sclerosis. Am J Manag Care 2013;19:S21-27.

15 Hannun YA, Obeid LM: Principles of bioactive lipid signalling: lessons from sphingolipids. Nature Rev 2008;9:139-150.

16 Zhang Y, Li X, Becker KA, Gulbins E: Ceramide-enriched membrane domains-structure and function. Biochim Biophys Acta 2009;1788:178-183.

17 Gulbins E, Bissonnette R, Mahboubi A, Martin S, Nishioka W, Brunner T, Baier G, Baier-Bitterlich G, Byrd C, Lang F, Kolesnick R, Altman A, Green D: FAS-induced apoptosis is mediated via a ceramide-initiated RAS signaling pathway. Immunity 1995;2:341-351.

18 Gulbins E, Li PL: Physiological and pathophysiological aspects of ceramide. Am J Physiol Regul Integr Comp Physiol 2006;290:R11-26.

19 Teichgräber V, Ulrich M, Endlich N, Riethmüller J, Wilker B, De Oliveira-Munding CC, van Heeckeren AM, Barr ML, von Kürthy G, Schmid KW, Weller M, Tümmler B, Lang F, Grassmé H, Döring G, Gulbins E: Ceramide accumulation mediates inflammation, cell death and infection susceptibility in cystic fibrosis. Nat Med 2008;14:382-391.

20 Becker KA, Riethmüller J, Lüth A, Döring G, Kleuser B, Gulbins E: Acid sphingomyelinase inhibitors normalize pulmonary ceramide and inflammation in cystic fibrosis. Am J Respir Cell Mol Biol 2009;42:716-724.

21 Bauer J, Liebisch G, Hofmann C, Huy C, Schmitz G, Obermeier F, Bock J: Lipid alterations in experimental murine colitis: role of ceramide and imipramine for matrix metalloproteinase-1 expression. PLoS One 2009;4:e7197. 
22 Grassme H, Jekle A, Riehle A, Schwarz H, Berger J, Sandhoff K, Kolesnick R, Gulbins E: CD95 signaling via ceramide-rich membrane rafts. J BiolChem 2001;276:20589-20596.

23 Gulbins E, Kolesnick R: Raft ceramide in molecular medicine. Oncogene 2003;22;7070-7077.

24 Steinman L, Zamvil SS: How to successfully apply animal studies in experimental allergic encephalomyelitis to research on multiple sclerosis. Annals Neurol 2006;60:12-21.

25 Agrawal S, Anderson P, Durbeej M, van Rooijen N, Ivars F, Opdenakker G, Sorokin LM: Dystroglycan is selectively cleaved at the parenchymal basement membrane at sites of leukocyte extravasation in experimental autoimmune encephalomyelitis. J Exp Med 2006;203:1007-1019.

26 Buhler LA, Samara R, Guzman E, Wilson CL, Krizanac-Bengez L, Janigro D, Ethell DW: Matrix metalloproteinase-7 facilitates immune access to the CNS in experimental autoimmune encephalomyelitis. BMC Neurosci2009;10:17.

27 Bauer M, Brakebusch C, Coisne C, Sixt M, Wekerle H, Engelhardt B, Fässler R: Beta1 integrins differentially control extravasation of inflammatory cell subsets into the CNS during autoimmunity. Proc Nat Acad Sci USA 2009;106:1920-1925.

28 Carpinteiro A, Becker KA, Japtok L, Hessler G, Keitsch S, Pozgajova M, Schmid KW, Adams C, Müller S, Kleuser B, Edwards MJ, Grassmé H, Helfrich I, Gulbins E: Regulation of hematogenous tumor metastasis by acid sphingomyelinase. EMBO Mol Med 2015;7:714-734.

29 Serrano D, Bhowmick T, Chadha R, Garnacho C, Muro S: Intercellular adhesion molecule 1 engagement modulates sphingomyelinase and ceramide, supporting uptake of drug carriers by the vascular endothelium. Arterioscler Thromb Vasc Biol 2012;32:1178-1185.

30 Rahn EJ, Iannitti T, Donahue RR, Taylor BK. Sex differences in a mouse model of multiple sclerosis: neuropathic pain behavior in females but not males and protection from neurological deficits during proestrus. Biol Sex Differ. 2014;5:4. 